\title{
'A coal face option': GPS' perspectives on the rise in antidepressant prescribing
}

\author{
Sara Macdonald, Jill Morrison, Margaret Maxwell, Rosalia Munoz-Arroyo,
} Andrew Power, Michael Smith, Matt Sutton, Philip Wilson

\section{ABSTRACT \\ Background}

Levels of antidepressant prescribing have dramatically increased in Western countries in the last two decades.

Aim

To explore GPs' views about, and explanations for, the increase in antidepressant prescribing in Scotland between 1995 and 2004 .

\section{Design}

Qualitative, interview study.

Setting

General practices, Scotland.

Participants

GPs in 30 practices $(n=63)$ purposively selected to reflect a range of practice characteristics and levels of antidepressant prescribing.

\section{Method}

Interviews with GPs were taped and transcribed. Analysis followed a Framework Approach.

\section{Results}

GPs offered a range of explanations for the rise in antidepressant prescribing in Scotland. Few doctors thought that the incidence of depression had increased, and many questioned the appropriateness of current levels of prescribing. A number of related factors were considered to have contributed to the increase. These included: the success of campaigns to raise awareness of depression; a willingness among patients to seek help; and the perceived safety of selective serotonin reuptake inhibitors, making it easier for GPs to manage depression in primary care. Many GPs believed that unhappiness, exacerbated by social deprivation and the breakdown of traditional social structures, was being 'medicalised' inappropriately.

\section{Conclusion}

Most antidepressant prescriptions in Scotland are issued by GPs, and current policy aims to reduce levels of prescribing. To meet this aim, GPs' prescribing behaviour needs to change. The findings suggest that GPs see themselves as responders to, rather than facilitators of, change and this has obvious implications for initiatives to reduce prescribing.

\section{Keywords}

depression; drugs; mental health; qualitative research; primary care.

\section{INTRODUCTION}

Levels of antidepressant prescribing have dramatically increased in Western countries in the last two decades. ${ }^{1-8}$ The reasons for this increase are not clear, but in Scotland they are not due to an increase in the incidence or prevalence of depression, or recorded help-seeking behaviour by patients. ${ }^{9}$ Most cases of depression in the UK are managed entirely in primary care and the vast majority of prescriptions for antidepressants are issued by GPs. ${ }^{10}$ GPs have, therefore, played a central role in the rise of antidepressant prescribing.

Concern about the risk of inadequate recognition and treatment of depression in the late 1980s and 1990s (addressed, for example, by The Defeat Depression Campaign ${ }^{11}$ ) is now matched by a

S Macdonald, BA, research fellow; J Morrison, PhD, FRCGP, professor of general practice; $\boldsymbol{P}$ Wilson, DPhil, FRCGP, senior clinical research fellow, Section of General Practice; $\boldsymbol{M}$ Smith, MRCGP, MRCPsych, consultant psychiatrist and senior research fellow, Section of Psychological Medicine, Division of Community Based Sciences, Faculty of Medicine, University of Glasgow, Glasgow. R Munoz-Arroyo, MSc, senior information analyst, Health Scotland, Edinburgh. A Power, FRCGP, FRCP, medical prescribing adviser, Prescribing Team, Pharmacy and Prescribing Support Unit, Victoria Infirmary, Glasgow. M Maxwell, PhD, reader, University of Stirling, Stirling. M Sutton, PhD, professor of health economics, Health Methodology Research Group, School of Community Based Medicine, University of Manchester, Manchester.

Address for correspondence

Sara Macdonald, Section of General Practice and Primary Care, Division of Community Based Sciences, Faculty of Medicine, University of Glasgow,

1 Horselethill Road, Glasgow G12 9LX.

E-mail: s.macdonald@clinmed.gla.ac.uk

Submitted: 22 April 2009; Editor's response: 4 June 2009; final acceptance: 2 July 2009.

() British Journal of General Practice

This is the full-length article of an abridged version published in print. Cite this article as: Br J Gen Pract 2009; DOI: 10.3399/bjgp09X454106. 
concern that antidepressants may be prescribed inappropriately. ${ }^{12}$ Doctors have been criticised for 'medicalising' social problems. ${ }^{13}$

There is large variation in prescribing antidepressants between practices, and the current authors have previously demonstrated that almost half of this variation between practices can be explained by population, GP, and practice characteristics. ${ }^{14}$ To understand better the increase and variation in antidepressant prescribing, the current researchers interviewed GPs about their management of depression, with a particular focus on antidepressant prescribing. This paper presents GPs' reflections on the reasons for the rise in antidepressant prescribing.

\section{METHODS}

\section{Sample}

All GP practices across Scotland were allocated to a sampling frame to facilitate purposive sampling. The frame consisted of a three-by-three matrix generated by two characteristics: (1) high, medium, and low levels of prescribing; and (2) high, medium, and low rates of increase in antidepressant prescribing. The aim was to recruit a purposive sample of 30 practices from across Scotland. The level of prescribing, using defined daily doses (DDDs), was calculated with data for financial year 2003/4, and the rate of increase in antidepressant prescribing was calculated comparing prescribing from 1995/6 with 2003/4. Prescribing data were accessed retrospectively from a national dataset of all prescriptions dispensed in Scotland and, as such, $2003 / 4$ was the most recent data available at the time of the study. One researcher carried out the practice selection. This ensured a mix of practices with respect to sex and age of partners, number of partners, size of practice, training status, deprivation, and rurality (Tables 1 and 2). The interviewer was blind to which cell of the matrix practices occupied.

As prescribing data are available only by GP practice and not by individual GP, it was appropriate that practices, rather than individual GPs, were recruited. At least half of the GPs in a practice had to agree to be involved for inclusion of their practice. Each GP in the practice was initially contacted by letter and followed up by telephone call within 2 weeks. GPs were given the option of individual, group, or telephone interviews.

\section{Qualitative interviews}

All interviews were conducted by one researcher during 2005 and 2006. The interviews were semistructured. A topic guide ensured that specific areas were covered but was flexible enough to allow additional themes to emerge. The topic guide

\section{How this fits in}

With limited access to psychological therapies, GPs face difficult decisions regarding whom to refer. Little is known as to how they make these decisions.

In the current qualitative study, GPs described themselves as considering a patient's wishes for and interest in referral, the patient's capacity to benefit from psychological therapy, and their own capacity to help the patient. They balanced likely effectiveness of psychological therapy for each patient with their own ability to help.

consisted of a series of key themes including 'possible explanations for the rise in antidepressant prescribing', 'depression management', and 'influences on prescribing'. Interviews lasted around 60 minutes and were taped and transcribed verbatim.

\section{Analysis}

Data analysis was inductive, continuous, and began from the start of data collection. While the analysis did not adhere strictly to a single analytic strategy, the method adopted in the Framework Approach ${ }^{15}$ was drawn on, meaning that the original aims of the

Table 1. Sampling frame: practice prescribing profile.

\begin{tabular}{lcc} 
Group in matrix $^{\mathrm{a}}$ & $\begin{array}{c}\text { Practices included } \\
\text { in study, } n\end{array}$ & $\begin{array}{c}\text { Total practices in } \\
\text { sampling frame, } n\end{array}$ \\
\hline Low prescribing/Low rate of increase & 5 & 42 \\
\hline Low prescribing/Medium rate of increase & 4 & 56 \\
\hline Low prescribing/High rate of increase & 0 & 1 \\
\hline Medium prescribing/Low rate of increase & 3 & 59 \\
\hline Medium prescribing/Medium rate of increase & 5 & 314 \\
\hline Medium prescribing/High rate of increase & 3 & 41 \\
\hline High prescribing/Low rate of increase & 1 & 6 \\
\hline High prescribing/Medium rate of increase & 3 & 45 \\
\hline High prescribing/High rate of increase & 6 & 23 \\
\hline
\end{tabular}

${ }^{a}$ Refers to the group allocated according to level and rate of increase of antidepressant prescribing; for example, Low prescribing/Low rate of prescribing denotes a low level of prescribing and a low level of increase in prescribing 1995/6 to 2003/4. A total of 587 practices in Scotland were assigned to the matrix according to prescribing levels. Not all practices in Scotland could be classified.

Table 2. Characteristics of participating practices.

Characteristics

\begin{tabular}{lccc}
\hline Partners, $n$ & Single-handed $=4$ & Medium $(2-3)=15$ & Large $(4+)=11$ \\
\hline Urban/rural, $n$ & Remote $=12$ & Small city $=2$ & Large city $=16$ \\
\hline Deprivation, $n$ & Deprived $=9$ & Medium $=14$ & Affluent $=7$ \\
\hline Sex, $n$ & $\begin{array}{c}\text { Predominantly } \\
\text { male }=14\end{array}$ & $\begin{array}{c}\text { Predominantly } \\
\text { female }=2\end{array}$ & $\begin{array}{c}\text { Roughly } \\
\text { equal }=14\end{array}$ \\
\hline Training practice, $n$ & Yes $=7$ & No $=23$ & - \\
\hline Ethnicity,$n$ & Yes $=5$ & No $=25$ & - \\
\hline
\end{tabular}

alf yes here, denotes more than half of partners in the practice completed their medical training outside the UK. 
study informed the analytic process but familiarisation with the data permitted other important themes to emerge. Data were indexed according to broad themes, and patterns both within, and across, data were identified. Interview transcripts were systematically managed using the QSR NVivo (version 2.0) data-handling package. Transcripts were read by two members of the research team and an analytic framework was developed after discussion of emergent themes.

\section{RESULTS}

\section{Participants}

Eighty-six practices were invited to take part in the study and 30 agreed. A total of 63 GPs ( 41 male and 22 female) were interviewed. As all practices had been entered into the sampling frame, selecting practices from all of the cells in the matrix ensured that a broad range of practices were involved (Tables 1 and 2). Telephone interviews were carried out with individual GPs in six practices and the remaining 24 practices opted for face-to-face interviews. Three practices preferred group interviews. These included two or three GPs and followed the traditional interview model, rather than a focus group.

\section{Prescribing trends}

Without exception, GPs recognised that there had been an increase in antidepressant prescribing since 1992, though few could predict the level of increase. Many GPs were surprised that prescribing levels had risen so steeply, particularly as they did not recognise a corresponding increase in the incidence or prevalence of depression. Most GPs believed that their own prescribing had increased over the same period, albeit to a lesser degree than the national average. ${ }^{16}$ Many felt uneasy about the increased use of antidepressants, although it was not always clear whether this uneasiness related to recent criticism of prescribing practice, or whether they thought that the level of prescribing was inherently 'wrong'.

When explaining the rise in prescribing, change was a constant theme. GPs did not see themselves as drivers of change, but instead as responding to external pressures. The changes they described will be considered in relation to three issues: recognition of depression, patient circumstances, and treatment of depression.

\section{Recognition of depression}

The core message of The Defeat Depression Campaign, launched in 1992 by the Royal College of Psychiatrists and the Royal College of General Practitioners (RCGP), was that depression was inadequately detected and treated in general practice. ${ }^{11}$ While few GPs interviewed mentioned this campaign unprompted, its impact was evident from their comments. Many acknowledged being more attuned to depressive symptoms, looking beyond somatic symptoms, and probing a little deeper if they suspected a patient might be experiencing depression. For many, the 'defeat depression' message was part of their GP training and emphasised the problematic nature of dealing with depression in practice. Although some felt that there had never been a problem with recognition, it is clear that the message has been accepted and normalised:

'There's a lot of em, you know there's a lot of pressure on us I think to not under diagnose or there has been in the last, whatever yeah I suppose 10 years, a sense that depression was around and was under diagnosed and under treated and yeah explicitly under treated and em ... it's the sort of a sense of a lot of harping on about "GPs don't do well with depression", "a lot of depression is unrecognised", or if recognised untreated so that's been there.' (practice [PR] 6, GP A)

'There was, in the early 90s and the 80s I think, there was a perception that there has been an under diagnosis of depression and under treatment of depression, and I think there was a lot of research that went on. There was a lot of stuff in the BMJ, general practice journals about screening tools to pick up depression and GPs were very much encouraged to look for depression, to look at the hidden agenda concept. Even if you look at the way the RCGP has worked with things like the video recording [as a training aid], things like that, it's all basically aimed at sort of going beyond "have you got a sore throat?".' (PR 11, GP A)

'GP training is more focused on the whole person not just their physical ailments and therefore there's more recognition that depression plays a very large part of the average GP workload on a day to day basis: both acute presentations and chronic maintenance of people with depression and mental health issues.' (PR 12, GP A)

'And we look for it, we tend to be more attune to people coming in, you know once upon a time maybe slip through the net because they would present with physical symptoms headaches all that kind of stuff and we might have just treated the headaches but actually nowadays we tend to be much more likely to say you know how are things generally, are there any stresses you know 
and I think that's probably why we are ... quicker at picking up depression than we used to be.' (PR 15, GP B)

'Some of it is past GP awareness, so I think there is an element of that, that we, you know maybe now we actively ask patients about their mood and you know and address those issues whereas maybe 10 or 15 years ago they may not have been seen as priority, they may not have been picked up so I think some of that is, you know, leads to the increase.' (PR 17, GP C)

The drive towards more active recognition and management of depression affected other professionals, besides GPs. GPs describe other members of the primary care team 'seeking out' depression with the overall result of a 'higher index of suspicion' in the community:

'Depression in the elderly, postnatal depression - they've been such big campaigns and screening things, you know. People are out there doing questionnaires and so health visitors referring are saying, you know "Postnatal", you know, eh "screening shows this woman's depressed", and there's more people coming in that way. Things like, we run screening clinics for cardiovascular disease, stroke this sort of thing, and part of the template for that is to run a screening procedure for anxiety and depression. Diabetes clinic, the nurse has to do a screening for anxiety/depression and so, I think it's [the increase in prescribing] inevitable.' (PR 2, GP B)

Many GPs felt that the stigma surrounding mental ill health had declined. GPs believed that patients are now more willing to present with, and seek help for symptoms of depression:

'I think the world has moved on. I mean I think these things like these sort of general government campaigns like, the ones that were on the telly last year about CBT - l've got mental illness and so on. I thought they were really good actually. So, I think the general idea is much more accepted. I think that's definitely one of the things that's moved on you know. The understanding and acceptance of mental illness is much, much better ... TV soap operas, people opening their hearts out on sort of TV or radio in interviews and stuff like that. All these sorts of things, like celebrities who have said they have had depression or drink or drug problems or whatever, all these things give the general public sort of permission to admit they have problems and to go and seek help.' (PR 17, GP A)

GPs reported that it is now more common for patients to self-diagnose depression and present to primary care simply for treatment. A number commented on the unique nature of depression, as a diagnosis patients 'demand'. GPs felt that this was tied to increased patient knowledge, particularly of antidepressant medication. A number were cynical about patient familiarity with 'depression' and many saw this as a negative consequence of raising its profile. Some talked about patients' misconceptions, believing that many see antidepressants as 'happypills' or 'lifestyle' drugs, offering a 'quick-fix'. This does not apply to all patients. GPs also recognised that some people would resist a diagnosis of depression and remain largely resistant to antidepressant medication.

\section{Patients' expectations}

GPs believe that many patients think that depression has a 'simple' solution that can be provided by their doctor. A causal relationship was described in relation to familiarity with the symptoms of depression, the increase in experience of depression in the community, and misconceptions about depression, leading to increased help-seeking. GPs talked of patients' expectations and felt that there are some people who assume that life will be continuously happy:

'I think that my parents' generation, who are all in their 80s, you know they came through the war and you know they weren't expecting to live the perfect life. I think that there are people now who are in their 30s and 40s whose expectations of the quality of their life is high and if it doesn't reach that height then they "go and get help". You know consumerism doesn't just mean being able to buy baked beans or colour televisions, it also means being able to buy health.' (PR 26, GP A)

Moreover, there was a suggestion from some that not only did patients have unrealistic expectations about life, but also that they were now unable to cope with normal life events:

'We all get stressed with things in life and we don't all need Prozac for that. I suppose that's part of my reluctance to prescribe, say to everybody who comes in who's a bit pissed off or grabs a box of tissues and bursts into tears "I need a prescription". You know, I wouldn't prescribe for somebody bereaved for example, not early on. You know bereavement's normal, you are meant to be upset, you know, and, yeah 
it's not nice and it's tough to get through but you know that doesn't mean you are depressed because it's normal to be upset ... and I can't cure that with a magic wand you know.' (PR 17, GP C)

\section{'Priests of yesteryear': the social role of GPs}

The majority of GPs described a social climate in which depressive symptoms flourished. Socioeconomic deprivation, family breakdown, the secularisation of society, increased debt, fear of crime, social isolation, and drug and alcohol misuse were all mentioned. Such social ills have caused community fragmentation, which has created a void that results in patients turning to GPs for help. Patients, GPs believe, are affording them an everexpanding role which was summed up by one GP who commented that they have become the 'priests of yesteryear'.

\section{Medicalising misery}

GPs thought that they were often the first or only professional to be consulted about the impact of personal and social problems on their patients' lives. This led GPs to question repeatedly whether they are treating depression or the effects of difficult or 'miserable' lives. The challenge was to differentiate between those patients who are genuinely depressed and those that are merely 'fed up', often with 'difficult' social circumstances. Some questioned the appropriateness of their response to these issues in practice:

'I think there is generally quite a lot of illness and morbidity out there because obviously a lot of people are unhappy rather than depressed and sometimes it's not easy to sort of work out if somebody's depressed or whether they are just unhappy with their life and, you know, got expectations from, you know, whatever media. So pressure is on expectations so yeah I certainly think there is a lot of just people that are unhappy and I think that's why antidepressants sometimes work and sometimes are less effective because it's just unhappy rather than depressed.' (PR 8, GP C)

'... people come along to see us with all sorts of problems that are not illnesses. People come in with unhappy relationships. They are unhappy at work. They have problems with their neighbours. They are generally dissatisfied with life and they expect the GPs to do something about this, which obviously we can't but we can label it as depression and medicate them. Whether that's actually doing anyone any good in the long run is arguable.' (PR 9, GP A)
'An antidepressant can't cure your social ills so if you are isolated, disabled, in chronic pain due to some physical ailment and perhaps with a depressive personality, then antidepressant might be the best thing to give you a lift: for some of them. But equally you get some people who are just glum by nature and no amount of antidepressant will help them. So it can be good for some things but it's not a panacea.' (PR 12, GP A)

'Well I think people get depressed because they are in unhappy situations, I still treat them for depression but unhappiness is part of it.' (PR 19, GP A)

'I don't think that's just as simple as medicalising unhappiness, probably you know a lot of the antidepressant prescribing we do it would be great to prescribe them a holiday or a job.' (PR 20, GP B)

GPs acknowledged that prescribing is only one option that is available for the management of depression but felt that other therapies were scarce and 'patchy'. Even though more appropriate interventions were often unavailable, GPs still needed to treat their patients. This led to reliance on what was described as the 'coal face option' of antidepressants. Many GPs were clearly frustrated with this situation:

'We've been hounded for so long that we were missing depression now we're, in a way, perhaps we're over diagnosing or perhaps we're treating more mild depression that in the past people would just have got on with. I mean, it then becomes quite subtle whether or not somebody's degree of unhappiness is tipping them into a mild depression or is it just life, and that's a bit of a fine line.' (PR 24, GP C)

\section{Selective serotonin reuptake inhibitors: the 'safer', 'coal face option'}

By far the single biggest change reported by GPs has been the advent of selective serotonin reuptake inhibitors (SSRIs). Established antidepressants, or 'old style' tricyclics, as they were often called, are now rarely used as first line treatment for depression. Many talked of the problems associated with tricyclics, particularly the unpleasant side effects and problems with overdose. One interviewee stated 'you had to have a pretty good reason to be on one'. Similar language was not used to describe SSRIs. There is no doubt that the majority saw SSRIs as safer, cleaner, cheaper drugs that can be tolerated by more patients: 
'I think because we have drugs now that are much cleaner drugs so that eh, we're not as limited in terms of the types of patients that we can prescribe for. Sometimes the old tricyclics people couldn't tolerate them ... ' (PR 7, GP D)

Few GPs made direct links between the availability of SSRI medication and the rise in prescribing rates. Nevertheless, the way in which they described the drugs, their confidence in prescribing them, and the almost total acceptance of their safety does suggest a causal relationship. The following quote hints that confidence in prescribing an SSRI could lead to over prescribing but also a practical solution:

'I don't think everybody who gets treated [with an SSRI] even in this practice or even by me necessarily would fulfil strict criteria, but it is one of these things that's relatively easy and pretty safe to do and is a coal face option.' (PR 8, GP A)

Some talked about living through 'fashions' in prescribing practice. They had noted recent negative publicity about SSRIs and felt that public opinion could now be turning against these drugs. Nevertheless, concerns about suicide and dependency were largely restricted to one specific SSRI (paroxetine). ${ }^{17}$ Other drugs continued to enjoy almost universal approval, and their safety was not questioned; although one GP did question the wisdom of life-time SSRI use. The perceived tolerability of SSRIs relative to tricyclics may have led to the view that they are not only safer but also 'milder' in their actions and side effects, thus allowing for the pharmacological treatment of 'milder' disease.

Contemporary thinking no longer makes a distinction between traditional concepts of 'endogenous' (biological) depression and 'reactive' (social) depression. Current prescribing guidelines ${ }^{18}$ emphasise the need to treat symptoms of depression, whether or not social or personal factors may have influenced an episode of low mood. However, some GPs discussed depression in terms of biological/endogenous or social/reactive depression. These doctors tended to be older, whereas younger GPs described depression in terms of severity, and focused on the treatment of symptoms rather than causes:

'I mean the old concept is about sort of reactive depression and non-reactive depression seem to be not quite so fashionable these days ... that seems to have gone out the window. I mean it used to be that somebody that was a so called endogenous depression you would be more likely to prescribe to than somebody who had a so called reactive depression, but those labels seem to be back out of fashion at the moment.' (PR 17, GP A)

'My impression is that truly, seriously, depressed people, I don't see any more of them. I think that's almost a separate illness. I see lots of unhappy folk with reactive depression.' (PR 8, GP B)

\section{Influence of guidelines}

Only one GP interviewed attributed the rise in antidepressant prescribing to adherence to best practice guidelines from the National Institute for Health and Clinical Excellence, namely, treating patients for a longer period of time; and another mentioned this among a series of possible explanations. GPs, however, typically reported prescribing for at least 6 months after the patient felt better, in line with guidelines. ${ }^{19}$

\section{Influence of pharmaceutical industry}

The impact of widespread tolerability and perceived safety of SSRIs on prescribing was only hinted at by a few. Others, however, emphasised the influence of the pharmaceutical industry at a macro level:

'I think there are also many groups of people who are just being bought off with prescriptions, just as we did with barbiturates and diazepam and so on and so forth and that's probably a fault of the system; we have a system that's only demand was controlled by doctors' time and so you're always going to have over easy prescribing given that you've got the pharma sitting in the background producing tabloid headlines like GPS over diagnose or under diagnose depression.' (PR 14, GP A)

GPs were asked directly about the potential influence of pharmaceutical companies. The vast majority reported that they 'did not see drug reps', although a few mentioned the power of advertising in medical journals. Nevertheless, when drug choice was discussed, some who dismissed the influence of 'reps' went on to talk about the prescription of nonformulary compliant drugs, which suggests that the advertising campaigns of the pharmaceutical industry may be more pervasive than GPs acknowledge.

\section{Universality of themes}

Practices were chosen to reflect the whole range of types of practices and behaviour in prescribing antidepressants. Although the interviewer was blind to the prescribing level and pattern in practices during the interviews, this information was available 
at analysis. This study compared transcripts from GPs in high prescribing practices and those in low prescribing practices but could find no evidence of a systematic difference in the comments made.

\section{DISCUSSION}

\section{Summary of main findings}

GPs in this study were aware of the increase in prescribing antidepressants and many were uneasy about it, although the drivers for the increase were considered to be largely external. These drivers included campaigns in the 1990s aimed at encouraging GPs to increase their recognition and active management of depression and, by reducing stigma, to encourage patients to present with symptoms of depression. Other explanations included a growing focus on 'good' mental health, encouraged by successive government campaigns to reduce stigma around mental ill health; the perceived trend towards the medicalisation of difficult lives coupled with a lack of availability of alternative management options; increasing social malaise; and the availability of SSRIs. It is not surprising that prescribing has increased, particularly as GPs have become better at diagnosing depression and because the drug treatments available are considered to be 'better'.

\section{Strengths and limitations of the study}

This study has been able to capture GPs' attitudes towards antidepressant prescribing. The focus was on general and collective prescribing behaviour rather than focusing on their actual prescribing behaviour with individual patients. This enabled some of the more controversial aspects of prescribing to be discussed openly.

The sampling frame ensured that experiences of GPs with all levels of prescribing were accessed. However, participation in the study was voluntary, and it is likely that GPs who were interested in mental health, comfortable with their prescribing practice, and prepared to reflect on this would be more likely to take part. Recruiting by practice ensured that a breadth of views were gained, but it also meant that some GPs who wanted to participate, particularly those in larger practices, could not do so if their colleagues did not wish to be involved.

\section{Comparison with existing literature}

Antidepressant prescribing in Scotland increased from 28.9 million DDDs in $1992 / 3$ to 128.3 million DDDs in 2004/5. ${ }^{20}$ Previous research has demonstrated that this is not due to an increase the presentation or recording of depression. ${ }^{9}$ In fact, consultations for depression have fallen significantly since 2003/4. ${ }^{21}$ This apparent contradiction may be partly explained by methods used to measure prescribing. GPs' sub-therapeutic prescribing (as was common with tricyclic drugs) is less possible with SSRIs, resulting in increased levels of DDDs of antidepressants prescribed without an increase in the number of patients being treated. ${ }^{22}$ In addition, GPs acknowledged that they are sometimes managing the impact of patients' difficult lives. Their difficulty in determining the boundaries of 'misery' and clinical depression might account for the lack of recording of such conditions as 'depression'.

The GPs interviewed believe that a range of outside pressures have influenced their prescribing, including the pastoral role in addition to the clinical role. It has been argued that GPs are now the 'default sources of comfort for issues such as bereavement, social isolation, and loneliness'. ${ }^{23}$ Other researchers have suggested a range of contributing factors; for example, the prescribing of antidepressants for other problems such as anxiety ${ }^{24}$ or prescribing at higher doses for longer duration as recommended in guidelines. ${ }^{19}$ In this study, guidelines were not mentioned by GPs as having a major influence, although most described prescribing behaviour, which indicated adherence to guidelines on duration of treatment.

GPs in this study felt that there had been an increase in the presentation of depression due to the success of campaigns to increase recognition and reduce stigma relating to mental health problems. For example, 'see me', the government-funded Scottish anti-stigma initiative, has conducted high profile media campaigns since 2002, and in 2003 the initiative reached an estimated $89 \%$ of the population through television advertising. ${ }^{25}$ Although early evaluations of The Defeat Depression Campaign demonstrated conflicting results, ${ }^{26-28}$ the Editor of the British Journal of General Practice commented in 1999 that 'what matters in the long term is how the messages of the campaign are taken up by individual practice teams and how quickly new research ... is taken up in day-to-day practice'. ${ }^{29}$ We believe that there is evidence from the current study that The Defeat Depression Campaign (strengthened by subsequent guidelines) has influenced practice. This view reflects the 'accumulation model of change' where the repetition of messages, particularly in respected journals, results in long-term change. ${ }^{30}$

This study did not find clear differences in views between GPs working in urban and rural settings, or between high and low prescribing doctors. Other studies have not shared this finding. ${ }^{31,32}$ This may be because GPs attribute a large part of the increase in prescribing to the need to support patients in difficult life circumstances. While such difficulties may be more prevalent in deprived practices, they are present 
across a range of practice settings. GPs collectively described the newer SSRIs as safer drugs that provided a 'coal face option' in the absence of alternatives. They expressed their discomfort at prescribing for what they felt were a complex mix of material disadvantage, early adversity, relationship issues, and maladaptive coping strategies. A perceived 'consumerism' in society was felt to lead to patients seeking medical/pharmaceutical help for problems with more complex personal and social origins. This is concordant with others' findings on GPs' emotional reactions to their prescribing and the discomfort they often feel ${ }^{33}$ when their only recourse is to prescribe medication while recognising more complex circumstances. ${ }^{34}$ The emotional conflict for GPs is exacerbated by their desire to help people in distress and the inadequate range of options open to them.

While managing the reality of the individual patient consultation, GPs in this study were keenly aware of the wider, societal concern surrounding the increase in prescription of antidepressants. Charges of ambivalence in prescribing decisions were rejected and this is confirmed by recent research that shows that GPs do not prescribe antidepressants without a clinical basis. ${ }^{35}$

\section{Implications for future research and clinical practice}

The Scottish government is concerned about the rate of increase in prescribing of antidepressants and has set a target to 'reduce the annual rate of increase of defined daily dose per capita of antidepressants to zero by $2009 / 10$ and put in place the required support framework to achieve $10 \%$ reduction in future years.' ${ }^{36}$ To achieve this, GPs require alternative management options for those patients whose 'mild' depression is not best managed with antidepressants. Use of severity when labelling depression can be unhelpful in primary care. The implication is that 'mild' depression poses less of a challenge to GPs, yet 'mild' often represents a complex picture of psychosocial difficulty and distress that fails to fit easily into 'symptom counting' definitions and is arguably harder to manage.

The process of change, in the context of GPs' antidepressant prescribing, will not happen overnight and will only occur with consistently reinforced evidence for change. The evidence base for 'appropriate' prescribing does not yet exist, but it is perhaps more important to pursue 'appropriate' management, which in many cases will include both antidepressants and 'talking therapies' for some, and self-help or alternative strategies for others. This may help to alleviate some of the concerns GPs expressed about their role, particularly in managing mild depression.
Health policy in Scotland is developing to support greater access to psychological therapies and improve access to better quality assessment for depression, as reflected in the current Integrated Care Pathway for Depression Guidelines. ${ }^{37}$ This policy direction is consistent with the problems identified by GPs in this study. Further work is required to investigate the potential for nonpharmacological approaches to support recovery from depression adequately. Given the scale of the problem, work will also be needed to review 'service delivery systems' with the capacity to cope with demand for help with depression in primary care.

\section{Funding body}

This study was funded by the Chief Scientist Office of the Scottish Executive Health Department (reference number: $\mathrm{CZH} / 4 / 198)$. This work is independent of the funder and does not necessarily represent its views

\section{Ethical approval}

This study was approved by Southampton A Multi-Centre Research Ethics Committee (reference number: 04/Q1702/53)

\section{Competing interests}

All authors have stated that there are none.

\section{Discuss this article}

Contribute and read comments about this article on the Discussion Forum: http://www.rcgp.org.uk/bjgp-discuss

\section{REFERENCES}

1. Paykel E, Brugha T, Fryers T. Size and burden of depressive disorders in Europe. Eur Neuropsychopharmacol 2005; 15(4): 411-423.

2. Hemels ME, Koren G, Einarson TR. Increased use of antidepressants in Canada: 1981-2000. Annals Pharmacother 2002; 36(9): 1375-1379.

3. Olfson M, Marcus SC, Druss B, et al. National trends in the outpatient treatment of depression. JAMA 2002; 287(2): 203-209.

4. Middleton N, Gunnell D, Whitley E, et al. Secular trends in antidepressant prescribing in the UK, 1975-1998. J Public Health Med 2001; 23(4): 262-267.

5. Reseland S, Bray I, Gunnell D. Relationship between antidepressant sales and secular trends in suicide rates in the Nordic countries. $\mathrm{Br}$ Psychiatry 2006; 188: 354-358.

6. Helgasson T, Tomasson H, Zoega T. Antidepressants and public health in Iceland. Time series analysis of national data. Br J Psychiatry 2004; 184: 157-162.

7. Guaiana G, Andretta M, Corbari L, et al. Antidepressant drug consumption and public health indicators in Italy, 1955 to 2000. J Clin Psychiatry 2005; 66(6): 750-755.

8. Van Marwijk H, Bijl D, Herman J, de Haan M. Antidepressant prescription for depression in general practice in the Netherlands. Pharm World Sci 2001; 23(2): 46-49.

9. Munoz-Arroyo R, Sutton M, Morrison J. Exploring potential explanations for the increase in antidepressant prescribing in Scotland using secondary analysis of routine data. Br J Gen Pract 2006; 56(527): 423-428.

10. Anderson IM, Nutt D, Deakin JFW. Evidence-based guidelines for treating depressive disorders with antidepressants: a revision of the 1993 British Association of Psychopharmacology guidelines. J Psychopharmaco 2000; 14(1): 3-20.

11. Management and Scientific Advisory Committee of the Defeat Depression Campaign. Defeat depression: the recognition and management of depression in general practice. London: Department of Health, 1993.

12. Heath I. Commentary: there must be limits to the medicalisation of human distress. BMJ 1999; 318(7181): 439-440.

13. Pilgrim D, Bentall R. The medicalisation of misery: a critical realist analysis of the concept of depression. J Mental Health 1999; 8(3): 261-274.

14. Morrison J, Anderson MJ, Sutton M, et al. Factors influencing variation in prescribing of antidepressants by general practices in 
Scotland. Br J Gen Pract 2009; 59(559): 25-31.

15. Ritchie J, Spencer L. Qualitative data analysis for applied policy research. In: Bryman A \& Burgess RG (eds). Analysing qualitative data. London: Routledge, 1994: 173-195.

16. Philp AV, Watson L, Muir R. Mental health in Scotland: information sources and selected insights. NHS Scotland Information and Statistics Division: Edinburgh, 2002.

17. World Health Organization. WHO Drug Information Vol. 17, No. 2 , 2003.

http://apps.who.int/medicinedocs/en/d/Js4954e/5.11.html\#Js4954e.5. 11 (accessed 7 Aug 2009).

18. Anderson IM, Nutt DJ, Deakin JF. Evidence-based guidelines for treating depressive disorders with antidepressants: a revision of the 1993 British Association for Psychopharmacology guidelines. J Psychopharmacol 2000; 14(1):3-20.

19. McIntosh A, Cohen A, Turnbull N, et al. Clinical guidelines and evidence review for panic disorder and generalised anxiety disorder. Sheffield: University of Sheffield/London: National Collaborating Centre for Primary Care, 2004.

http://guidance.nice.org.uk/CG22/guidance/pdf/English (accessed 30 Jul 2009)

20. Morrison J, Anderson MJ, Donald SM, et al. Relationship between antidepressant and anxiolytic/hypnotic prescribing in Scotland: a mixed methods study. Eur J Gen Pract 2008; 14(3\&4): 129-135.

21. ISD Scotland. General Practice - Practice Team Information (PTI). Depression: number of patients consulting. Edinburgh: ISD Scotland, 2009. http://www.isdscotland.org/isd/3711.html (accessed 7 Aug 2009).

22. NHS Quality Improvement Scotland. Clinical Indicators 2007. Edinburgh: NHS Quality Improvement Scotland, 2007. http://www.nhshealthquality.org/nhsqis/3802.html (accessed 7 Aug 2009).

23. Pink J, Jacobson L, Pritchard M. The 21st century GP: physician and priest? Br J Gen Pract 2007; 57(543): 840-842.

24. Hollinghurst S, Kessler D, Peters TJ, Gunnell D. Opportunity cost of antidepressant prescribing in England: analysis if routine data. $B M$ J 2005; 330(7498): 339-361.

25. See me campaign. See me so far: a review of the first 4 years of the Scottish anti-stigma campaign. Edinburgh: See me campaign. http://www.seemescotland.org.uk/seemesofar/seemesofar2 (accessed 27 Jul 2009).

26. Orrell M, Baldwin B, Collins E, Katona C. The impact of the Defeat Depression Campaign. Psychiatric Bulletin 1996; 20: 50-51.

27. Macaskill A, Macaskill ND, Nichol A. The Defeat Depression Campaign: amid-point evaluation of its impact on general practitioners. Psychiatric Bulletin 1997; 21: 148-150.

28. Rix S, Paykel ES, Lelliott P, et al. Impact of a national campaign on GP education: an evaluation of the Defeat Depression Campaign. $\mathrm{Br}$ J Gen Pract 1999; 49(439): 99-102.

29. Wright AF. Through a glass darkly: understanding depression. $\mathrm{Br} \mathrm{J}$ Gen Pract 1999; 49(439): 91-92.

30. Armstrong D, Reyburn H, Jones R. A study of general practitioners' reasons for changing their prescribing behaviour. BMJ 1996; 312(7306): 949-952

31. Prosser H, Almond S, Walley T. Influences on GPs' decision to prescribe new drugs: the importance of who says what. Fam Pract 2003; 20(1): 61-68.

32. Jaye C, Tilyard M. A qualitative comparative investigation of variation in general practitioners' prescribing patterns. Br J Gen Pract 2002; 52(478): 381-386.

33. Henriksen K, Hansen EH. The threatened self: general practitioners self-perception in relation to prescribing medicine. Soc Sci Med 2004; 59(1): 47-55.

34. Maxwell M. Women's and doctors' accounts of their experiences of depression in primary care: the influence of social and moral reasoning on patients' and doctors' decisions. Chronic Illn 2005; 1(1): 61-71.

35. Cameron I, Reid I, Lawton K. Appropriateness of antidepressant prescribing: an observational study in a Scottish primary-care setting. Br J Gen Pract 2009; 59(566): 644-649.

36. The Scottish Government. HEAT Targets. Edinburgh: The Scottish Government, 2008.

http://www.scotland.gov.uk/Topics/Health/health/mentalhealth/servicespolicy/DFMH/antidepressantprescribing (accessed 3 Aug 2009)

37. Scottish Executive. Delivering for mental health. Edinburgh: The Scottish Executive, 2006

http://www.scotland.gov.uk/Publications/2006/11/30164829/0 (accessed 30 Jul 2009). 